\title{
One day-ahead prognosis of energy demand using artificial intelligence and biometeorological indices
}

\author{
Zafirakis D. ${ }^{{ }^{*}}$ Moustris K.P. $^{2}$, Alamo D.H. ${ }^{3}$, Nebot Medina R.J. ${ }^{3}$, \\ 1 Soft Energy Applications \& Environmental Protection Lab, Mechanical Engineering \\ Department, Technological Education Institute of Piraeus, 250 Thivon and P. Ralli Str., GR- \\ 12244 Athens, Greece \\ 2 Laboratory of Fluid Mechanics, Mechanical Engineering Department, Technological \\ Education Institute of Piraeus, 250 Thivon and P. Ralli Str., GR-12244 Athens, Greece \\ 3 Instituto Tecnológico de Canarias S.A., Cebrián 3, 35003 Las Palmas de Gran Canaria, \\ Spain \\ *corresponding author e-mail: dzaf@teipir.gr
}

\begin{abstract}
Nowadays demand side management has become an important issue. Managing the energy resources in an optimal manner has become imperative among energy planners and policy makers. An integrated energy management approach is essential for the sustainable development of any electricity grid. The main objective of this work is the development of a forecasting model in order to predict one day ahead the energy demand of Tilos Island, Greece. For this purpose, an artificial neural network (ANN) forecasting model was developed to predict the energy demand of the entire island region. Prediction concerns 24-hours ahead on an hourly basis, with the developed ANN model being fed with historical data of energy demand, historical data of solar irradiation and historical data of a biometeorological index known as Cooling Power Index. Results show that the proposed methodology gives a sufficient forecast of energy demand in order to design an automated energy demand information tool for end-users such as distribution and transmission system operators.
\end{abstract}

\section{Introduction}

During the recent years, increased interest is recorded in the field of smart grids (Malik and Lehtonen 2016, Tuballa and Abundo 2016) and in the development of novel demand side management (DSM) techniques (Haider et al. 2016, Stathopoulos et al. 2014) that can facilitate the latter operation. One of the essential elements for the effective implementation of such techniques is the ability to reliably forecast load demand several hours ahead (Pérez-García and Moral-Carcedo 2016). In 
fact, there is a trade-off between the forecasting ability of prognostic tools and the level at which DSM is implemented, meaning that increased forecasting reliability several hours ahead comes also with moderate interventions in terms of loads' control and vice versa.

At the same time, it can be argued that the smaller the scale of the grid under examination, the more challenging are both the performance of load forecasting tools and the level of DSM application (Neves et al. 2016). This links to increased probability of having sudden changes of load demand, which defines local load demand as sensitive to considerable variation. Especially for small scale island regions, this can be also justified by the fact that industrial loads are normally absent and thus base load operation depends almost exclusively to residential demand as well as to pumping needs for irrigation purposes. On the other hand, linking load demand with residential alone needs comes with the benefit of interpreting energy consumption through human comfort needs. This is also the case for the small island of Tilos, in SE Greece, featuring a population of $\sim 500$ people, where average load demand does not exceed $200 \mathrm{~kW}$, with the respective annual peak load and total consumption reaching $1 \mathrm{MW}$ and $3 \mathrm{GWh}$. Considering the challenging aspects of load demand prognosis at the level of small scale island grids and the fact that a smart microgrid is under development on the island of Tilos, Greece, an artificial neural network was developed in the present work so as to forecast the energy demand (ED) of Tilos up to 24-hours ahead.

\section{Data and methodology}

ANN are a branch of artificial intelligence developed in the 1950s aiming at imitating the biological brain architecture. They are parallel-distributed systems made of many interconnected nonlinear processing elements (PEs), called neurons (McCulloh and Pitts 1943, Hect-Nielsen 1990). A renewal of interest has grown exponentially in the last decades, mainly for the availability of suitable hardware that has made them convenient for fast data analysis and information processing.

In this work, an ANN forecasting model known as Multilayer Perceptron (MLP) was developed to predict the energy demand (ED) of the entire Tilos island region. MLP is the most commonly used type of ANN topology. Its structure consists of processing elements (PEs) and connections. The PEs called neurons are arranged in layers, the input layer, one or more hidden layers, and the output layer (Hect-Nielsen 1990, Moustris et al. 2010, Nastos et al. 2011).

For the appropriate training of the developed ANN mean hourly values of wind speed, air temperature, global solar irradiation and energy demand were used. These meteorological data have been calculated by one minute step records. More specifically, a meteorological mast was installed in the specific location measuring the above meteorological parameters every minute. Furthermore, the ED was measured every ten minutes by two dedicated grid load meters covering the entire 
and half the island respectively. The collected data cover the time period from 05/04/2015 up to 13/12/2015 (253 days).

Initially, the hourly values of wind speed and air temperature were calculated and from these values the hourly values of a biometeorological index known as Cooling Power (CP) were extracted (Nastos et al. 2011). Then, a data file with the available mean hourly values consisting of 6072 cases (253 days x 24 hours) was shaped. This file contains the input and output data for the training of the developed ANN. Table 1 presents the input and output data which were used for the training of the developed ANN model.

Table 1. Description of input and output data.

\begin{tabular}{ll}
\hline & The number of the month $(4,5,6, \ldots, 12)$ \\
& The number of the hour $(0,1,2, \ldots, 23)$ \\
& The number of the day (Monday=1 up to Sunday=7) \\
& The type of the day $(0=$ Sundays and holidays, $1=$ typical working day) \\
& The global solar irradiation $\left(\mathrm{kWh} / \mathrm{m}^{2}\right)$ from the 24 previous hours \\
& The CP values $\left(\mathrm{W} / \mathrm{m}^{2}\right)$ from the 24 previous hours \\
& The ED $(\mathrm{kWh})$ from the 24 previous hours \\
\hline Output data & The ED $(\mathrm{kWh}) 24$ hours ahead in hourly bases $(24$ output values simultaneously)
\end{tabular}

For the appropriate ANN training the initial data file was separated in three subsets. The first subset consists $60 \%$ of the available data and was used for ANN training. The second subset consists (randomly selected group of data) $20 \%$ of the available data and was used for the cross validation test and the third subset consists of the rest (randomly selected group of data) 20\% of the available data and was used for the testing phase in order the forecasting accuracy of the developed ANN model to be examined. For the evaluation of the forecasting ability of the proposed load demand methodology appropriate statistical evaluation indices were applied (Moustris et al. 2010, Nastos et al. 2011).

\section{Results}

The developed ANN model forecasts the ED 24-hours ahead on an hourly basis using only historical meteorological and ED data from the previous 24hours. In this way, the developed model is a dynamic model that updates the forecasting every hour. Figure 1a,b depicts the best prediction of ED (4-hours ahead prognosis) and Figure 1c,d presents the worst prediction of ED (19-hours ahead prognosis). It seems that in both cases the prognosis is fairly adequate for such a magnitude of ED.

In order for the forecasting ability of the proposed ANN model to be examined in greater detail, the values of RMSE, MBE, $\mathrm{R}^{2}$ and IA were calculated for every 
forecasting hour ahead (1-24). Figure $2 a, b$ depicts the values and the trends of the aforementioned evaluation statistical indices. Figure $2 \mathrm{c}$ shows the diurnal variation (typical day) of ED during the warm (May-September) and the cold (OctoberApril) season of the year.

Figure $2 \mathrm{a}$ shows that RMSE ranges between $37.2 \mathrm{~kW}$ and $69.3 \mathrm{~kW}$ and MBE ranges between $+19.2 \mathrm{~kW}$ and $+48.1 \mathrm{~kW}$. In any case MBE is positive which indicates that the proposed ANN model overestimates the ED of the next hours. The biggest error (for both RMSE and MBE) appears during the $19^{\text {th }}$ hour ahead (worst prediction-Figure $1 \mathrm{c}, \mathrm{d}$ ) and the smallest error is presented at the $4^{\text {th }}$ hour ahead (best prediction-Figure $1 \mathrm{a}, \mathrm{b})$. Concerning the coefficient of determination $\left(\mathrm{R}^{2}\right)$ it is ranging between 0.859 and 0.947 which means that in any case the model is able to explain at least $85.9 \%$ up to $94.7 \%$ of ED variance. Additionally, IA is ranging between 0.896 and 0.972 (the closest to the unit the best the prediction is) indicating that the predicted ED values are very close to the corresponding observed ED values. Finally, in comparison with Figure $2 c$, it seems that the developed ANN forecasting model has captured the pattern of the diurnal ED variation (typical day).

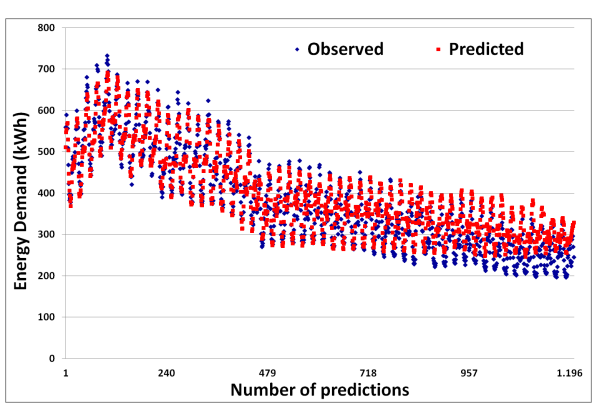

(a)

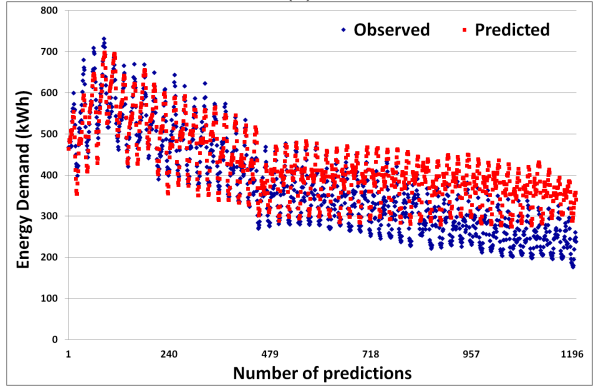

(c)

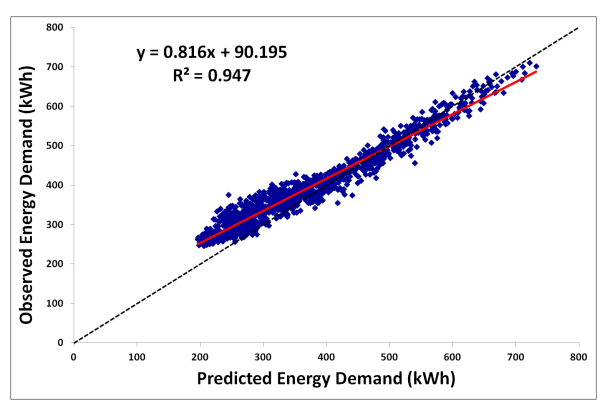

(b)

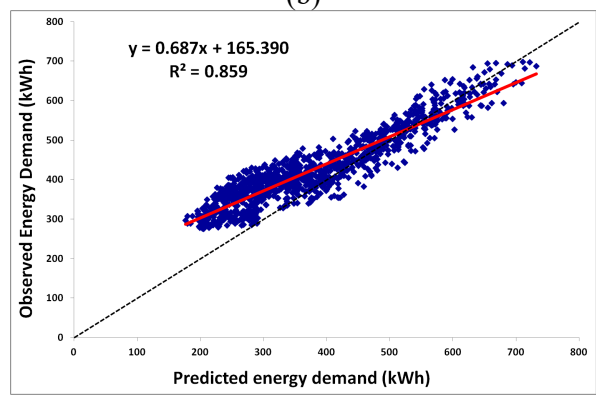

(d)

Fig. 1. Observed vs Predicted mean hourly load demand values 4 hours ahead (a) and 19 hours ahead (c). Scaterrplots between the Observed and Predicted mean hourly load demand values 4 hours ahead (b) and 19 hours ahead (d). 


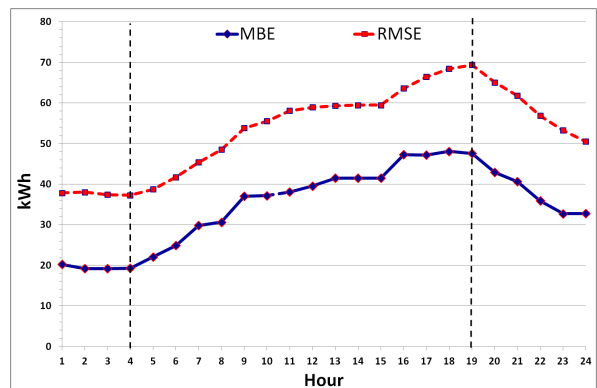

(a)

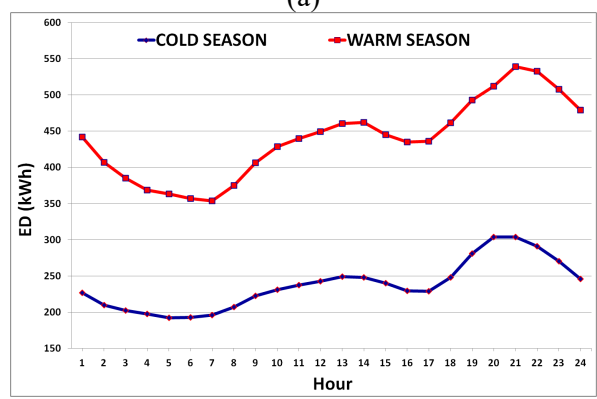

(C)

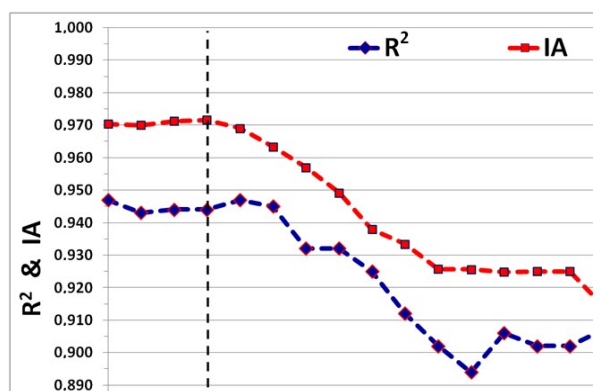

(b)

Fig. 2. Values and trends of the evaluation statistical indices for the 24 hours of prognosis (a) and (b). Energy demand typical daily profile for the cold and the warm season of the year (c). Period 2001-2013.

\section{Conclusions}

The aim of this work was the development of a prognostic tool in order to forecast the ED of the Island of Tilos, Greece, 24-hours ahead, on an hourly basis. For this purpose, an ANN forecasting model was developed. Results showed that the forecasting ability of the proposed model is remarkable. More specifically, all the appropriate evaluation statistical indices that have been used to evaluate the forecasting ability of the developed model showed that the prediction of ED is very good and sufficient at a statistical significant level of $\mathrm{p}<0.01$.

\section{Acknowledgment}

This work took place and was funded under the project TILOS (Horizon 2020 Low Carbon Energy Local / small-scale storage LCE-08-2014). This project has received funding from the European Union's Horizon 2020 research and innovation programme under Grant Agreement No 646529.

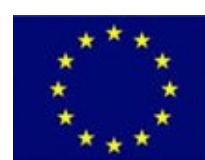




\section{References}

Haider HT, See OH, Elmenreich W (2016) A review of residential demand response of smart grid. Renew Sust Energ Rev 59:166-178.

Hect-Nielsen R (1990). Neurocomputing. Reading, M.A: Addison-Wesley.

Malik FH, Lehtonen M (2016) A review: Agents in smart grids. Electr Pow Syst Res 131:71-79.

McCulloh WS, Pitts W (1943) A logical calculus of ideas immanent in Nervous activity. B Math Biophys 5:115-133.

Moustris KP, Ziomas IC, Paliatsos AG (2010) 3-Day-Ahead Forecasting of Regional Pollution Index for the Pollutants $\mathrm{NO} 2, \mathrm{CO}, \mathrm{SO} 2$, and $\mathrm{O} 3$ Using Artificial Neural Networks in Athens, Greece. Water Air Soil Poll 200:29-43.

Nastos P, Moustris K, Larissi I, Paliatsos A (2011) Air Quality and Bioclimatic Conditions within the Greater Athens Area, Greece-Development and Applications of Artificial Neural Networks. Advanced Air Pollution, InTech-Open Access (ISBN: 978-953-307-511-2):557-584.

Neves D, Brito MC, Silva CA (2016) Impact of solar and wind forecast uncertainties on demand response of isolated microgrids. Renew Energ 87:1003-1015.

Pérez-García J, Moral-Carcedo J (2016) Analysis and long term forecasting of electricity demand through a decomposition model: A case study for Spain. Energy 97:127-143.

Stathopoulos M, Zafirakis D, Kavadias K, Kaldellis JK (2014) The Role of Residential Loadmanagement in the Support of RES- Based Power Generation in Remote Electricity Grids. Energy Procedia 46:281-286.

Tuballa ML, Abundo ML (2016) A review of the development of Smart Grid technologies. Renew Sust Energ Rev 59:710-725. 